\title{
Sound Watermarking Utilizing Spread Spectrum
}

\author{
Komal Goswami \\ M.Tech (CSE) \\ Ward no.4 Suraj Kund Road \\ Kangra(H.P)
}

\author{
Nitika Sharma \\ Asst. Professor \\ Sri Sai University \\ Palampur(H.P)
}

\begin{abstract}
With the new headway in the PC and telecom to store, delivered and disperse media, records over the globe turn out to be simple. However the possession and copyright of these are generally not ensured. Computerized watermarking plans have been considered as a mean of ensuring these documents. The calculations for watermarking propose so far work just for computerized picture and video. Exploration work in light of spread range procedure for watermark implanting. The calculation needn't bother with the first sound transporter signal for removing watermark; the first flag is utilized just for correlation with the installed sound. The Results show that the watermark installing calculation is capable of being heard as well as more strong against the assaults like separating and pressure. Examinations have demonstrated that the unintelligibility and vigor execution objectives can be accomplished.
\end{abstract}

\section{Keywords}

Digital Signal Processing, Watermarking Process, Audio Watermark

\section{INTRODUCTION}

Amid couple of years Digital watermarking assume an imperative part in copyright assurance. This is because of the progressions in PC and telecom field which make simple to store, appropriate and get to the $\mathrm{mm}$ documents over the globe. So to shield these documents from unapproved access is must .Digital watermarking is characterized as "the act of intangibly changing a Work [the image] to implant a message about that Work." (see figure 1)

It can be seen from the expressed definition that watermarking is firmly identified with steganography, the craft of disguised correspondence. However which means of watermarking varies marginally from the means of steganography [1]. In steganography the concealed message has no connection to the medium in which the message is transmitted. Where as in watermarking the concealed message is constantly about the medium in which it is transmitted.

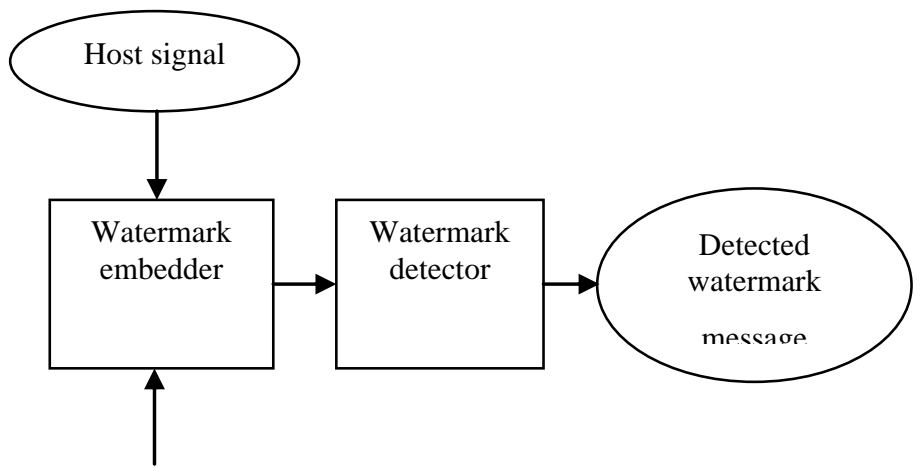

Watermark Signal

Figure 1 an overview of the general watermarking system
A decent sound watermarking calculation ought to fulfill the accompanying necessities:

a) Fidelity: it alludes the watermark ought not influence unique sound sign after the watermark is inserted into it.

b) b) Robustness: it is the capacity of watermarking calculation to survive when different assaults will perform on it.

c) c) Detection blunders: it is computed by two means false positive and false negative. In False positive locator calculation distinguishes a watermark when there is no watermark present, while in false positive it doesn't recognize notwithstanding when it is available. Calculation ought to have the capacity to keep from these sorts of mistakes.

\section{RELATED WORK}

Quantities of watermarking procedures have been proposed so far to make a vigorous sound watermarking system.

A Novel Spread Spectrum Digital Audio Watermarking [1] Spread range method is a standout amongst the most imperative procedures for concealing mystery data into a sound sign. Spreading nature of this strategy makes the discovery of implanted data is verging on unimaginable for pernicious client. There are two executions of this method: either utilizing direct grouping spread range methodology or recurrence jumping spread range. In the immediate arrangement spread range methodology, Exclusive-OR operation is utilized for spreading the implanted data while in the recurrence using so as to jump spread range procedure, spreading is performed the bouncing succession. An advanced sound watermarking plan in light of spread range strategy to install the watermark. This technique does not require the first sound bearer signal when separating watermark utilizing the visually impaired extraction sound watermark.

DWT method using LSB coding[2] Another watermarking framework DWT system utilizing LSB coding for copyright insurance of computerized substance and clamor concealment strategy for watermark recuperation. In watermarking framework, the first sound is fragmented by DWT change that can be executed utilizing LSB coding. Through the DWT technique sound sign changes to DWT coefficient that are in the two sections first is the thick area and second one is the rarefaction district.

Spread-spectrum watermarking of audio signals [3] The security ramifications of the created systems and audit watermark heartiness on a benchmark suite that incorporates a blend of sound preparing primitives including: time-and recurrence scaling with goodness and-vacillate, added substance and multiplicative commotion, Re-testing, Requantization, clamor diminishment, and separating. A promising answer for this issue is denoting the media signal with a mystery, hearty and intangible watermark (WM). The 
media player at the customer side can recognize this imprint and thus uphold comparing e-trade arrangement.

\section{Mixing-attack-proof Randomized Embedding Audio Watermarking System [4]}

Sound blending extraordinarily corrupts the watermarking security. Subsequently, it is vital to present another procedure known as sound blending assault verification sound watermarking calculations. Initially, adjusted regulation is acquainted with dispose of the host signal obstruction and enhance the connection score solidness. A various synchronization system which at the same time synchronizes diverse blended segments is additionally used to further enhance the synchronization execution .Mixing assault evidence sound watermarking calculation demonstrates an in number level safety to blending assaults and it can oppose the blending assault with up to 5 blended segments. To ensure the survival of the shrouded data, watermark ought not to be deleted after some sound operations or a few assaults, which is the principle worry of watermarking security.
Blind audio watermarking for tamper detection based on LSB [5] Blind sound watermarking for temper identification in view of LSB ought to distinguish any treating and demonstrate the temper area. This new improves strategy consolidated DCT, FFT and DWT routines and accordingly presents another calculation that gives an in number association between sound vector individuals.

\section{PROPOSED WORK}

Nonetheless, numerous compelling advanced picture and video watermarking calculations have been proposed and actualized at a business scale. In any case, because of the way that the human sound framework is much more perplexing and touchy than the human visual framework, couple of calculations have been proposed for sound watermarking. Our go for adding to a powerful technique for sound watermarking as shown in figure 2 .

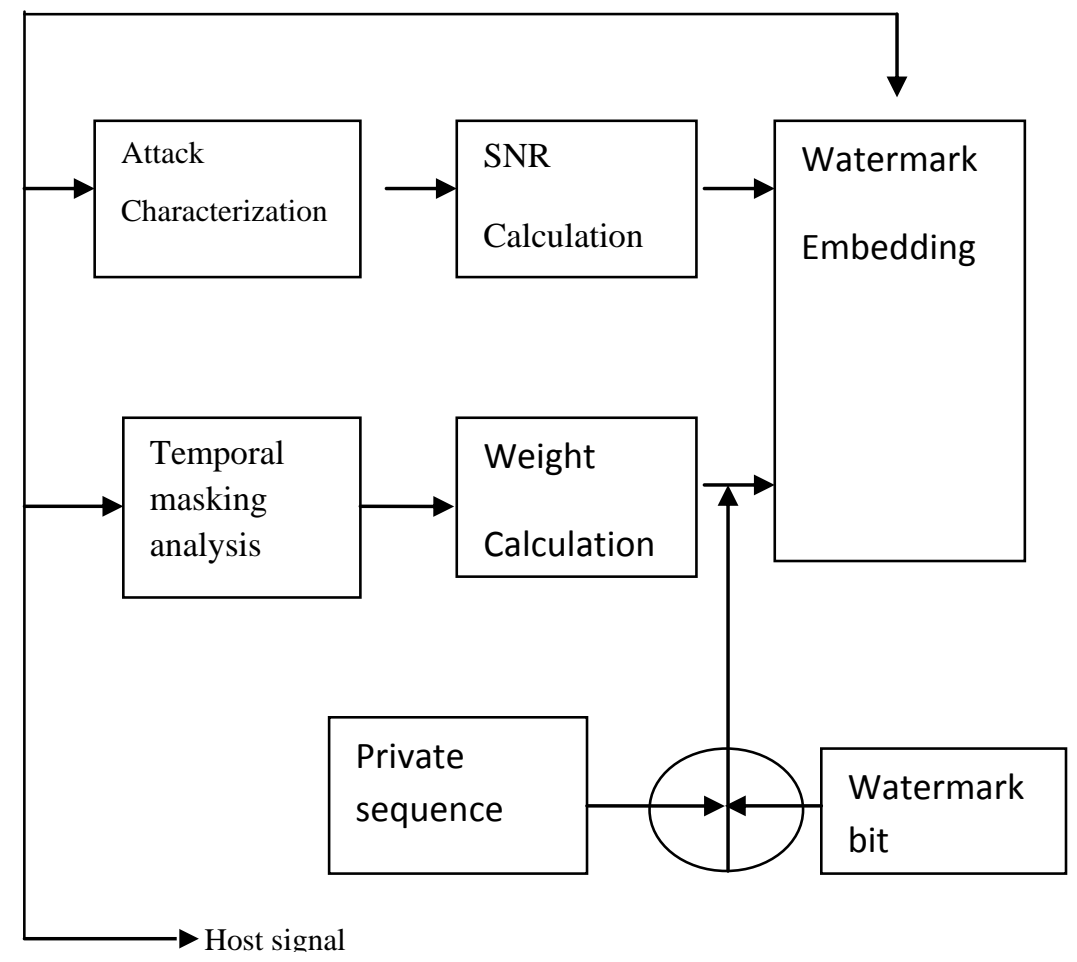

Figure 2 proposed audio watermarking embedding scheme

\section{RESULT AND ANALYSIS}

The Audio watermarking procedures sound square of 8820 bits at once, this is done to upgrade expansive network operations in matlab. Inexact length of time of square is $0.2 \mathrm{~s}$. The procedure begins watermarking after 30 pieces, wm_peak $=2$ compares to $5 \mathrm{~Hz}$; underneath the recurrence scope of capable of being heard range. It can be modified for some other frequencies(see figure 3). The enhancement component for the watermarked unearthly esteem ought to be kept between 10-15, 10 being the ideal worth. 
Watermark Embedding Process

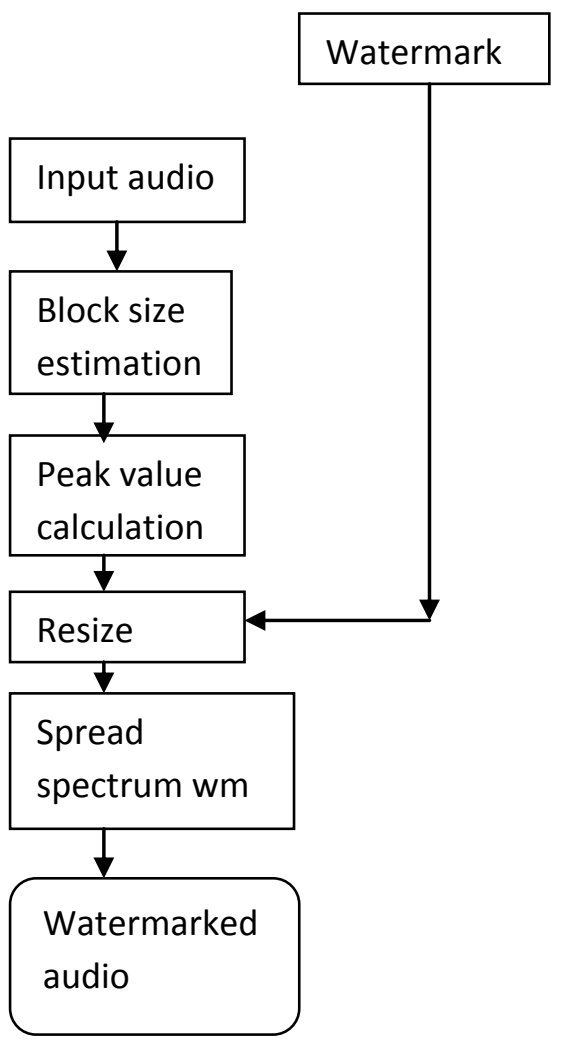

Figure 3 audio watermarking process

Watermark Detection process

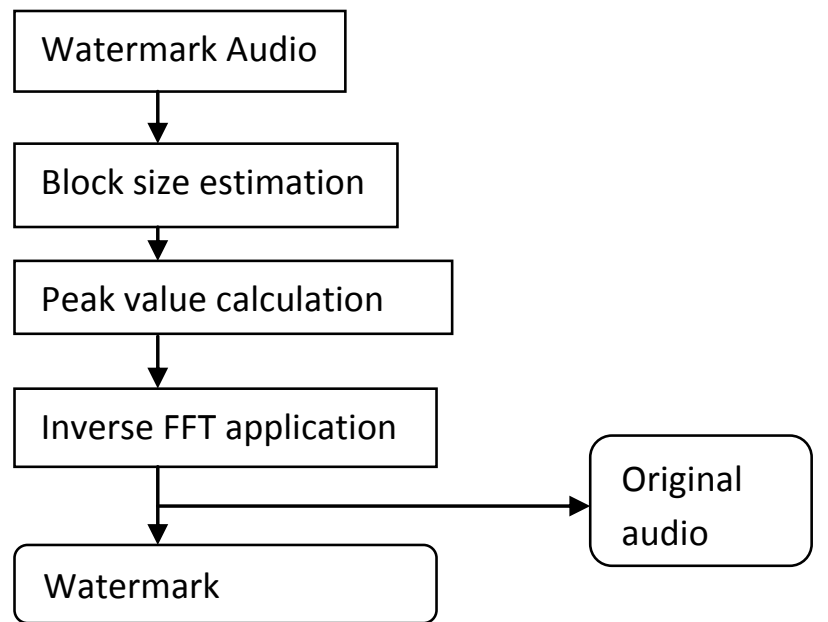

Figure 4 spread spectrum watermark detection processSpectrum Analysis

The investigation of electrical signs, also called signal examination, is a major test for all intents and purposes all electronic configuration specialists and researchers(see figure 4). While it gives significant understanding into a signal's properties, signal investigation is just on a par with the instrument with which it is performed. Range analyzers and vector signal analyzers are two instruments usually utilized to investigate electrical signs as shown in figure 6,7,8,9. Mp3 files are being compressed and the related peaks are made as per the watermark is embedded(see figure 10,11).

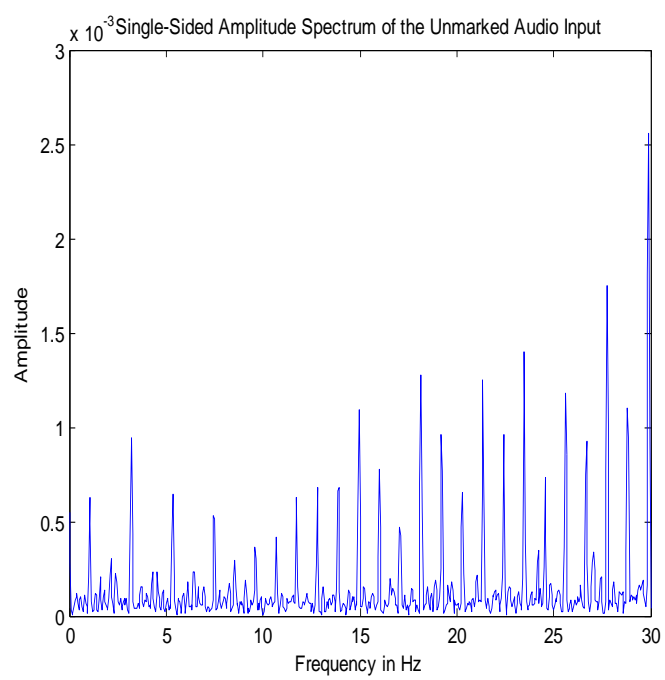

Figure 5 single sided amplitude spectrum of the unmarked audio file

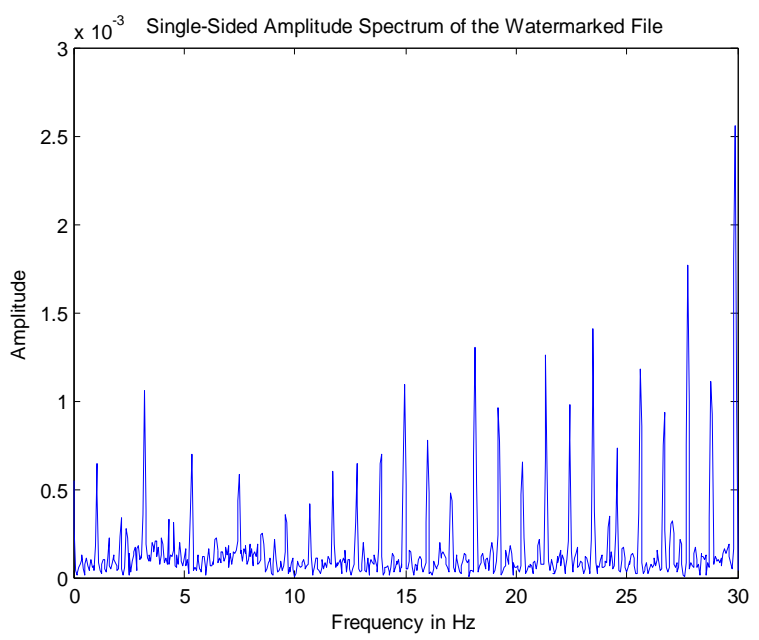

Figure 6 single sided amplitude spectrum of the water marked audio file

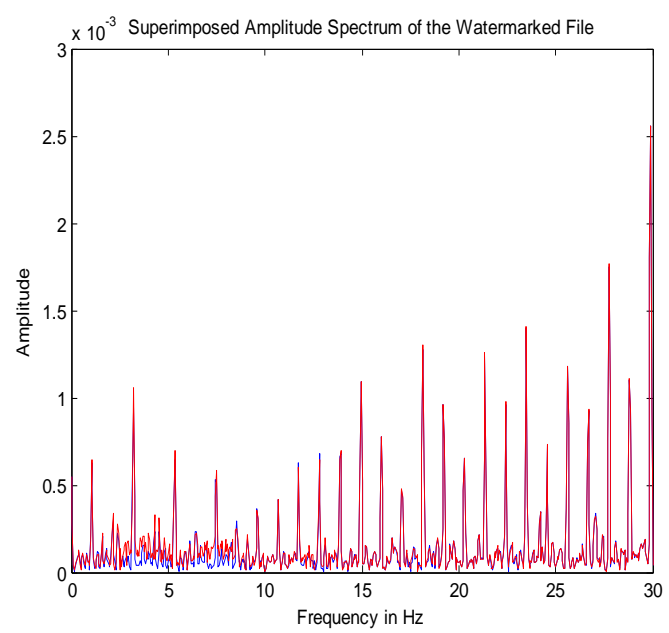

Figure 7 single sided amplitude spectrum of the water marked superimposed over audio file 


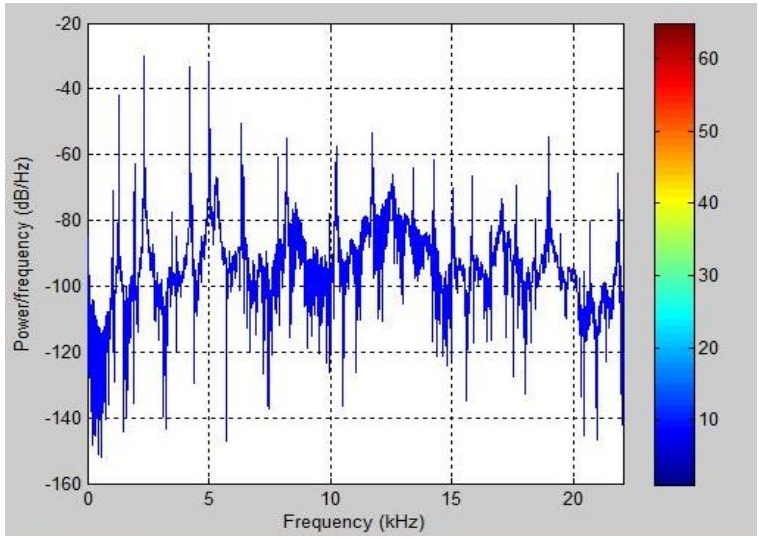

Figure 8 Frequency vs Power user per frequency in the Audio Spectrum

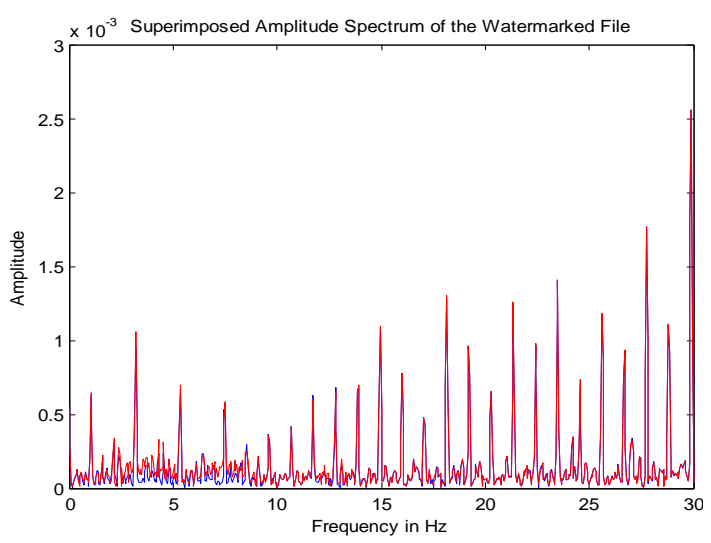

Figure 9 Single sided amplitude spectrum of the watermarked superimposed over audio file

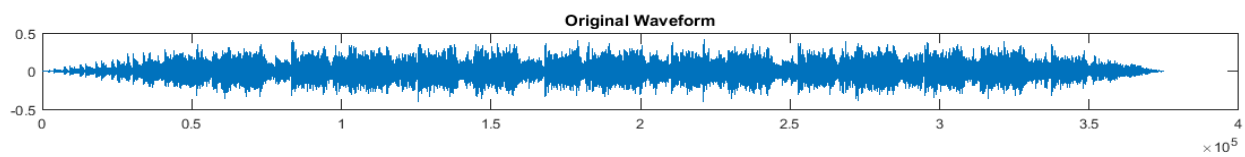

Compression Factor 2
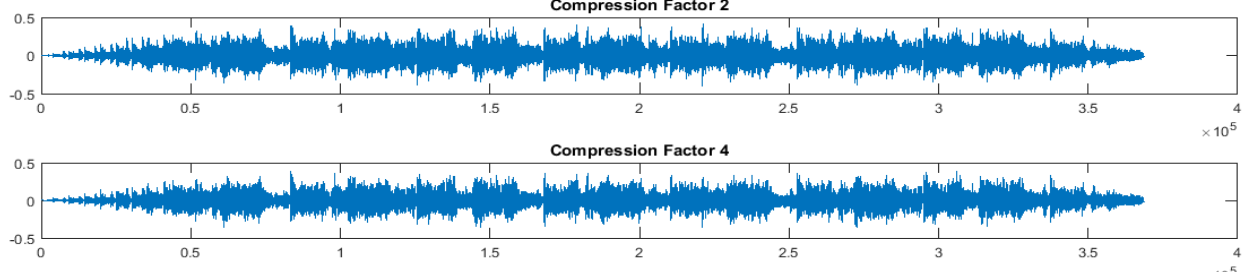

Compression Factor 8

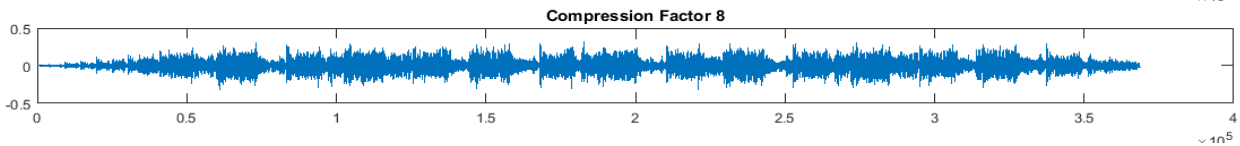

Figure 10Mp3Compressed
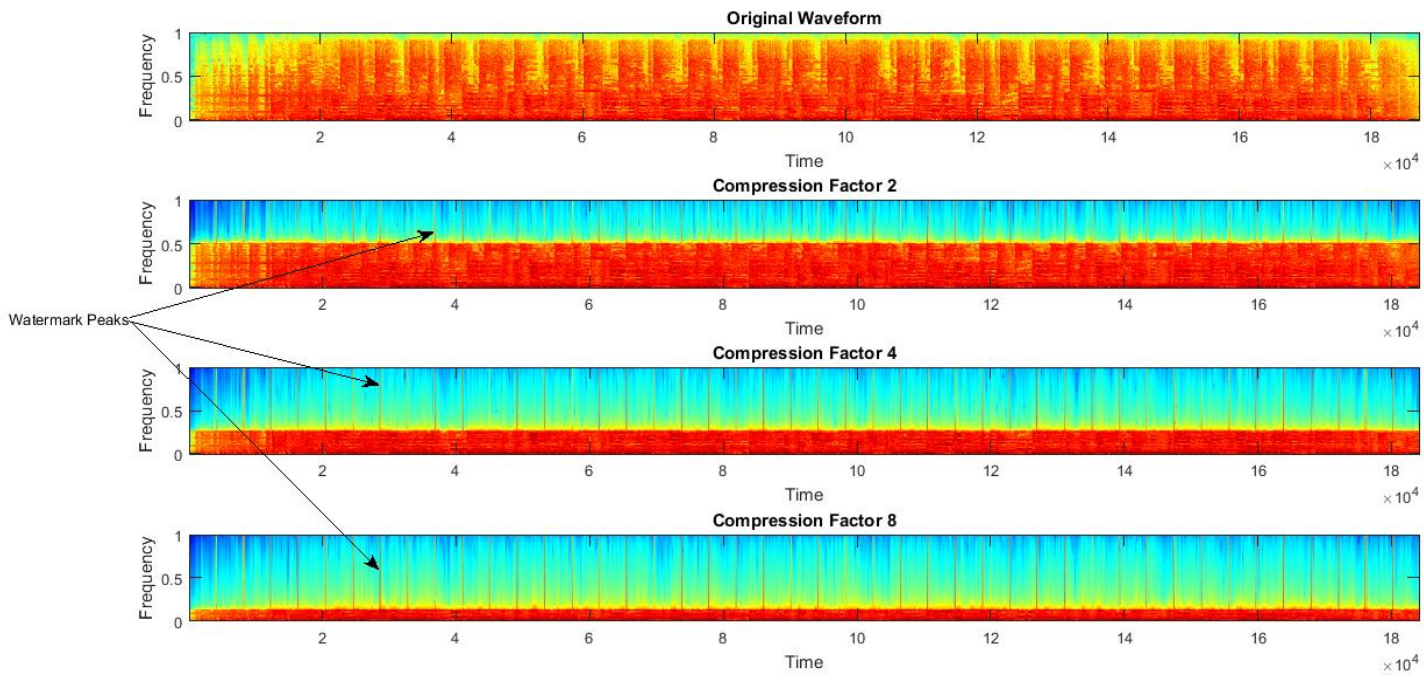

Figure11 Watermark peak

\section{CONCLUSION AND FUTURE SCOPE}

We have demonstrated that the proposed procedure gives different upgrades over conventional Spread Spectrum Watermarking, ordinary watermark installing time is under 5 sec, and recuperation time is even lower around $2.3 \mathrm{sec}$ all things considered for info test taken. The proposed watermarking procedure can be promptly connected to essentially any sound flag as of now utilizing Spread Spectrum, which exploits the increases. Additionally, the technique does not require any adjustment in the identification plan (de-inserting), and at times it could be connected even to frameworks that are as of now being utilized the new proposed system can further enhance the nature of sign. 


\section{REFERENCES}

[1] Can, Yekta Said, et al . "A Novel Spread Spectrum Digital Audio Watermarking." (2014).

[2] lal Verma, Mr Munna, and Miss Lakhwinder Kaur. "An Efficient Audio Watermark Embedding \& Extraction DWT Method with Noise Removing Technique." (2013).

[3] Kirovski, Darko, and Henrique S. Malvar. "Spreadspectrum watermarking of audio signals." Signal Processing, IEEE Transactions on 51, no. 4 (2003): 1020-1033
[4] Guo, Yuhong, Qingsheng Yuan, Xuemin Zhao, Jian Liu, and Yonghong Yan. "Mixing-attack-proof Randomized Embedding Audio Watermarking System." Journal of Computers 8, no. 12 (2013).

[5] Ghobadi, Alireza, A. Boroujerdizadeh, A. H. Yaribakht, and Roozbeh Karimi. "Blind audio watermarking for tamper detection based on LSB." In Advanced Communication Technology (ICACT), 2013 15th International Conference on, pp. 1077-1082. IEEE, 2013 